\title{
Transient Stability Assessment using Decision Trees and Fuzzy Logic Techniques
}

\author{
A. Y. Abdelaziz, Dr., Prof. \\ Electrical Power \& Machines Dept. Faculty of Engineering, Ain Shams University, Cairo, Egypt \\ E-mail: almoatazabdelaziz@hotmail.com \\ M. A. El-Dessouki, Dr. Assistant Prof. \\ Electrical Power \& Machines Dept. Faculty of Engineering, Ain Shams University, Cairo, Egypt \\ E-mail: mdessuki@hotmail.com
}

\begin{abstract}
Many techniques are used for Transient Stability assessment (TSA) of synchronous generators encompassing traditional time domain state numerical integration, Lyapunov based methods, probabilistic approaches and Artificial Intelligence (AI) techniques like pattern recognition and artificial neural networks.

This paper examines another two proposed artificial intelligence techniques to tackle the transient stability problem. The first technique is based on the Inductive Inference Reasoning (IIR) approach which belongs to a particular family of machine learning from examples. The second presents a simple fuzzy logic classifier system for TSA. Not only steady state but transient attributes are used for transient stability estimation so as to reflect machine dynamics and network changes due to faults.

The two techniques are tested on a standard test power system. The performance evaluation demonstrated satisfactory results in early detection of machine instability. The advantage of the two techniques is that they are straightforward and simple for on-line implementation.
\end{abstract}

Index Terms - Transient Stability, Artificial Intelligence, Inductive Inference, Decision Trees, Fuzzy Logic

\section{Introduction}

Various types of disturbance in the power network can lead to oscillations in power flows, voltages, generator phase angles, etc. In severe cases it is possible for one or more generators to loose synchronism and being to rotate at a different speed which leads to damage to generator plant. Transient stability assessment examines the capability of power system to return back to an acceptable steady-state condition after a large disturbance.

TSA has been examined by many researches [1, 2, 3] the traditional numerical integration method [1] discretizes the machine swing equations to obtain the evolution with time of the machine rotor angles but it is tedious and not suitable for on-line environment. Lyapunov stability theory of non-linear systems was used in [2]. Even though these methods are accurate, they are computationally tedious. In [3] a probabilistic assessment of TS for a multi-machine power system was suggested and random nature of the fault type and location were considered in details.

Recently, Artificial Intelligence (AI) has been applied to power system and improved the solutions in many areas. The pattern recognition methodology was used in the TS problem by some authors [4, 5]. In [4], different classifiers like the Bayes Quadratic classifier, Polynomial discriminant method and Nearest Neighbour decision rule were used for fast security assessment. The K-means clustering pattern recognition technique was also used in out-of-step detection [5].

The authors of [6] discussed the evaluation of the effectiveness of neural networks for rapid determination of critical clearing times for practical networks with varying outages and load patterns. They concluded that neural networks have difficulty in returning consistently accurate answers under varying network conditions using only steady state attributes.

In [7], an out-of-step prediction approach based on neural networks with improving the classification performance was presented. The suggested scheme is characterized by simple structure based only on local measurements.

The work presented in [8] was the first using the Inductive Inference Reasoning (IIR) approach for TSA. In [9], the decision tree (DT) transient stability method was introduced via a case study carried out on the French EHV power system, but the disadvantage of [8, 9] is that the attributes used to represent the operating points were simple pre-fault variables like bus voltages, active and reactive power injections. Authors of [10] demonstrated how DT can be constructed off-line and then utilized on-line for predicting TS in real time using post-fault synchronized phasor measurements. 
In [11], the induction and abduction methodologies of AI were utilized to develop the logic for a smart relay with early detection of system instabilities. Authors of [12] showed how to use DT for predicting loss of synchronism on an AC intertie using apparent resistance along with its rate of change measured near the electrical center of the intertie. Response based control by a DT is achieved by turning every time sample from every simulation into an input-output pair for the DT training set.

In [13], a new method for eigenvalue region prediction of critical stability modes of power systems based on decision trees was presented. The paper proved that DT's are fast, easy to grow, and provide high accuracy for eigenvalue region prediction. A genetic algorithm is implemented to search for the best set of inputs providing the highest performance in stability assessment.

From the other side, the fuzzy sets theory is also employed in TSA problem because it can take into account qualitative information about transient stability that remains unutilized by conventional approaches to the problem (human operators stability judging under imprecision). It can also provide the margin of system stability under a given operating condition. The application of fuzzy concepts in TSA was introduced for the first time in [14], where an index providing an evaluation of the system level of security by considering the values of generator accelerations and kinetic energies after the occurrence of a large disturbance was presented.

The authors of [15] presented a fuzzy logic classifier for TSA using three variables (load condition, fault location and clearing time) as inputs of the fuzzy classifier. The low correct classification percentage obtained $(92 \%)$ was due to the nature of the input variables. A predictive out-of-step relaying approach using fuzzy rule-based classification was presented in [16]. The advantage of this approach is that it does not need any telemetery equipment since it uses an active power transducer fed from local measurements on the generator terminals to produce the input features to the fuzzy system.

In [17], a class of fuzzy hyper-rectangular composite neural networks which utilize real-time phasor angle measurements to provide fast transient stability prediction for use with high speed control was developed. From the simulation tests on a sample power system, it reveals that the proposed tool can yield a highly successful prediction rate in real-time.

The work in [18] presented a methodology to analyze transient stability using a neural network based on ART architecture (adaptive resonance theory), named fuzzy ARTARTMAP neural network for real time applications. The security margin is used as a stability analysis criterion, considering three-phase short circuit faults with a transmission line outage.
The proposed fuzzy ART-ARTMAP neural network has a superior performance, in terms of precision and speed, when compared to conventional ARTMAP, and much more when compared to the neural networks that are trained by conventional back propagation algorithm.

Authors of [19] presented fuzzy-probabilistic modeling techniques for system component outage parameters and load curves. Based on the fuzzyprobabilistic models, a hybrid method of fuzzy set and Monte Carlo simulation for power system risk assessment was proposed to capture both randomness and fuzziness of loads and component outage parameters.

This paper presents two AI methods for TSA based on the inductive inference reasoning approach and the fuzzy logic approach. The two methods have been applied to a small standard test power system to demonstrate their validity. Compared with recent approaches, the two methods presented in this paper are straightforward, accurate, simple for real-time implementation and suitable to be applied for large power systems.

\section{Principle of the Inductive Interference Resonating Approach}

The inductive inference reasoning method pertains to the domain of artificial intelligence, more particularly, the family of machine learning from examples [20]. Inductive inference reasoning method builds decision rules off-line which are automatically designed in the form of Decision Trees (DTs) built in a top down fashion. According to the method, a DT is built on the basis of a pre analyzed training set, composed of states or operating points. Each of these states is represented by a group of attributes which are variables characterize the condition of the machine in question. These attributes are guessed to have a significant influence on the machine behavior. The detection of the transient stability condition involves traversing the DT for the given operating condition.

A DT classifies each input vector according to a series of tests. The diagram of a DT is flow chart in the shape of an upside-down tree as shown in Fig. 1. Starting at the top node, the flow branches right or left depend on the outcome of a simple test. For numerical data, the test is whether a particular element of the input vector exceeds a threshold. Processing proceeds down the tree until a terminal node is reached. The input is classified according to the class of the terminal node.

Even though the inductive inference reasoning method chooses the best attributes among the given attributes, it is unable to determine whether a combination of these attributes would give better results. It is also intuitively understood that the quality of the training set, with respect to states of system behavior on occurrence of the contingency, has 
considerable effect on the reliability of the DT constructed. Hence, the choice of attributes and development of a representative training set are very crucial for the IIR method.

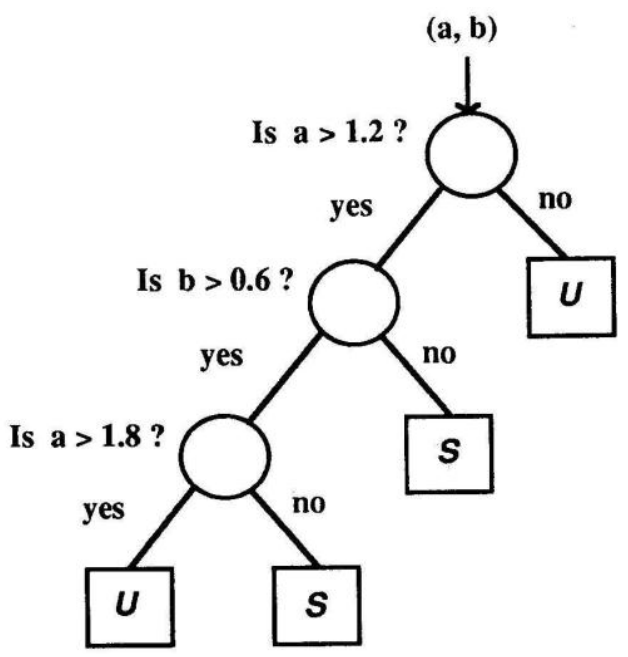

Fig. 1: Simple Decision Tree for Illustration Purposes

\section{Fuzzy Sets and Fuzzy Sets Operations}

Recently the publications on applications of fuzzy set theory to power systems has been increased which indicates its potential role in dealing with power systems problems. Although the obtained results are promising, fuzzy set theory is not widely accepted. The reasons are: misunderstanding of the concept, excessive claims of some researchers, lack of implemented and available systems, and its status as a new theory. Fuzzy set theory has been given more attention due to its achievements made in applications to other areas and it is felt that there is a need to develop more information on this subject [21].

Fuzzy logic system estimates functions from sample data. It is a model free estimator, which estimates a function without requiring a mathematical description of how the output functionally depends on the input. It learns the model only from samples. A fuzzy logical system is much closer in spirit to human thinking and natural language than traditional logic systems.

A fuzzy set $F$ in a universe $U$ is characterized by a membership function $\mu_{\mathrm{F}}$ which takes values in the interval $[0,1]$ namely, $\mu_{\mathrm{F}}: U \rightarrow[0,1]$. Thus a fuzzy set $F$ in $U$ is represented as a set of ordered pairs of generic element $u$ and its grade of membership function:

$$
\mathrm{F}=\left\{\left(\mathrm{u}, \mu_{\mathrm{F}}(\mathrm{u})\right) \mathrm{u} \mid \mathrm{C} \quad \mathrm{U}\right\}
$$

Let $A$ and $B$ be two fuzzy sets in $U$ with membership function $\mu_{A}$ and $\mu_{B}$ respectively. The fuzzy set operations of union, intersection and complement for fuzzy sets are defined via their membership functions.
Union: The membership function $\mu_{\mathrm{AUB}}$ of the union AUB is pointwise defined for all $u \in U$ by

$$
\mu_{\mathrm{AUB}} \quad(\mathrm{u})=\max \left[\mu_{\mathrm{A}}(\mathrm{u}), \mu_{\mathrm{B}}(\mathrm{u})\right]
$$

Intersection: The membership function $\mu_{\mathrm{A} \cap \mathrm{B}}$ of the intersection $A \cap B$ is pointwise defined for all $u \in U$ by

$$
\mu_{\mathrm{A} \cap \mathrm{B}}(\mathrm{u})=\min [\mu \mathrm{A}(\mathrm{u}), \mu \mathrm{B}(\mathrm{u})]
$$

Complement: The membership function $\mu \overline{\mathrm{A}}$ of the complement of a fuzzy set $\mathrm{A}$ is pointwise defined for all u $\mathrm{C} U$ by

$$
\mu_{\overline{\mathrm{A}}}(\mathrm{u})=1-\mu_{\mathrm{A}}(\mathrm{u})
$$

The components of conventional and fuzzy systems are quite alike, differing mainly in that fuzzy system contain "fuzzifiers" which convert inputs to their fuzzy representations, and "defuzzifiers" which convert the output of the fuzzy process logic into "crisp" (numerically precise) solution variables. The basic configuration of fuzzy logic control or classifier system is shown in Fig. 2.

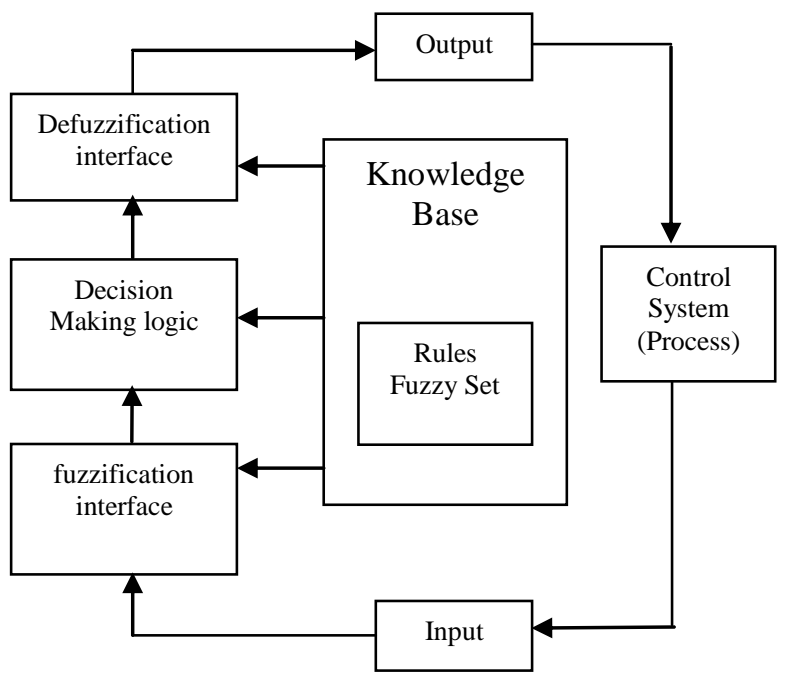

Fig. 2: The Basic Configuration of Fuzzy Logic Control

The fuzzification interface measures the values of input variables and then converts input data into suitable linguistic values which may be viewed as label of fuzzy sets.

The knowledge base comprises knowledge of the application domain and the attendant control goals. It consists of a "data base" and a "linguistic fuzzy control rule base". The database provides necessary definitions, which are used to define linguistic control rules and fuzzy data manipulation. The rule base characterizes the control goals and control policy by means of a set of linguistic control rules. 
The decision-making logic is the core of the operation. It has the capability of simulating human decision-making based on fuzzy logic concepts and the capability of inferring fuzzy control actions employing fuzzy implication and the rules of inference in fuzzy logic. The inference mechanisms in the fuzzy operation are generally much simpler than those used in atypical expert system. There are two principal methods of inference in fuzzy systems: The Min-Max method and the fuzzy additive method.

Deffuzification is the final phase of fuzzy reasoning. The defuzzification interface convertsthe range of values of output variables to yield a non-fuzzy control action. Defuzzification uses the centroid or center of gravity technique to find the "balance" point of the solution by calculating the weighted mean of the fuzzy region. For fuzzy solution A, the centroid is formulated as follows:

$$
\text { c.o.g }=\frac{\sum_{i=0}^{n} d_{i} \mu_{A}\left(d_{i}\right)}{\sum_{i=0}^{n} \mu_{A}\left(d_{i}\right)}
$$

where $d$ is the $i^{\text {th }}$ domain value and $\mu_{\mathrm{A}}(\mathrm{di})$ is the truth membership value for that domain point [22].

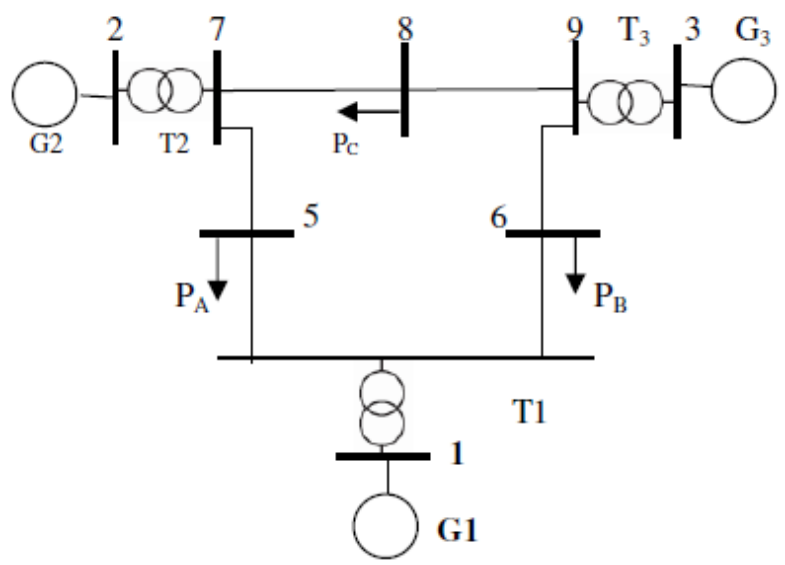

Fig. 3: Single Line Diagram of Power System under Study

\section{Application of the Inductive Inference Approach to Transient Stability Problem}

In this section, the method and the procedures for application of the inductive inference reasoning approach to the transient stability problem are described. The example system under study is a nine bus, three generators standard test power system proposed by Western System Coordinating Council in Canada. A one line diagram for the system is shown in Fig. (3) and the system characteristics are given in [23]. For this system, pre-fault and fault-on attributes are selected. A training set is generated which is further used in the DT construction. Cases, for which stability has to be determined, are then generated in the same way as the training set. These cases are then applied to the DT to determine their stability class. The percentage misclassification of these cases gives the reliability of the DT.

\subsection{Selection of Attributes}

There are many important quantities which have significant indication of machine stability and as a result their pattern would dominate the pattern of stable and unstable classes. The choice of candidate attributes is directed by the following heuristics:

1) The transient stability behavior is a strong function of the local variables i.e. the variables which are electrically close to the fault location.

2) The transient stability behavior can not be completely understood by considering pre-fault or static variables. Dynamic variables like initial accelerations of machine are representative as they inherently reflect machine dynamics and the changes in the network topology. Chiefly three types of attributes have been considered as follows:

\section{Type I:}

- Simple pre-fault attributes like bus voltages, line flows, rotor internal angles, and mechanical power input to generators.

- Heuristic pre-fault attributes like mean, minimal and maximal bus voltages, total active and reactive power load.

\section{Type II:}

- Simple fault-on attributes like machines speeds, accelerations at inception of fault and at instant of fault clearing.

- Heuristic fault-on attributes like the kinetic energy deviation at instant of fault clearing and mean machine accelerations.

\section{Type III:}

A combination of the two types I \& II.

The Decision Trees for each of the attributes types have been constructed and their reliability has been evaluated.

\subsection{Generation of Training Set Samples}

This is an important off-line task of the IIR algorithm. The quality of the training set determines the reliability of the DT for unseen cases. Two factors have been considered when building the training set. 
- Transient stability depends on the total system load level.

- For any load level, transient stability behavior differs with different distributions of loads and generations.

Therefore, generation of the training set samples should fulfill the previous two factors. It is assumed that the loads are randomly distributed and they have a normal distribution shape with the following means:

$$
\left[\mathrm{P}_{\mathrm{A}}, \mathrm{P}_{\mathrm{B}}, \mathrm{P}_{\mathrm{C}}\right]=[1.25,0.9,1.0] \text { p.u. }
$$

For load flow analysis, Bus 1 is taken as the swing bus and Buses 2 and 3 are voltage controlled buses with voltage magnitude of 1.025 p.u. For each load sample, the loading of the generators is determined by economical dispatch of the total load among generators, followed by a load flow analysis. A three phase short circuit is assumed to occur at one line very close to one of the buses of the system and the fault is removed by tripping out the faulted line. The Runge-Kutta numerical integration approach is applied to find the class of each sample. A sample is classified as unstable if the rotor angle of the critical generator which is chosen to be G3 reaches 180 degrees within one second [4], otherwise the sample is classified as stable.

Generation of samples is performed by both of the changing of the fault location and the loading conditions of the system prior to the occurrence of the fault. A group of samples is generated at six different fault locations with three different load levels (1.6, 1.0, 0.4) p.u. for each of the three loads of the power network under study and the clearing time is taken 150 ms. This results in 162 samples.

Number of stable samples $=146(90 \%)$.

Number of unstable samples $=16(10 \%)$.

The fault locations are:

$\begin{array}{ccccccc}\text { Bus number } & 4 & 5 & 6 & 7 & 8 & 9 \\ \text { Faulted line } & 4-6 & 5-4 & 6-4 & 7-5 & 8-9 & 9-6\end{array}$

In order to improve the classification performance, a normalization process is performed to all the variables of the training set.

\subsection{The Chosen Attributes}

\section{Type I:}

- Active power injections at buses 6,8 and 9 .

- Reactive power injections at buses 6,8 and 9.

- Mechanical input power of the critical generator.

- Total active power load.

- Total reactive power load.
- Voltage of each bus.

- Mean of bus voltages.

- Pre-fault internal angle of the critical generator.

\section{Type II:}

- Reactive power at the instant after fault inception.

- Machines speeds at the instant of fault clearing.

- Machines accelerations at the instant after fault inception.

- Machines accelerations at instant of fault clearing.

- The kinetic energy deviation of the critical generator at the instant of the fault clearing.

- The average (mean) accelerations of the generators.

Type I attributes are direct outcome of the load flow results. The other attributes are determined from a transient stability program using the second-order model of the machine.

\subsection{The Chosen Attributes:}

The decision tree with Types I, II and III attributes are shown in Figs. (4), (5) and (6) respectively. The shown DTs are chosen after extensive trials and they represent the best results obtained.

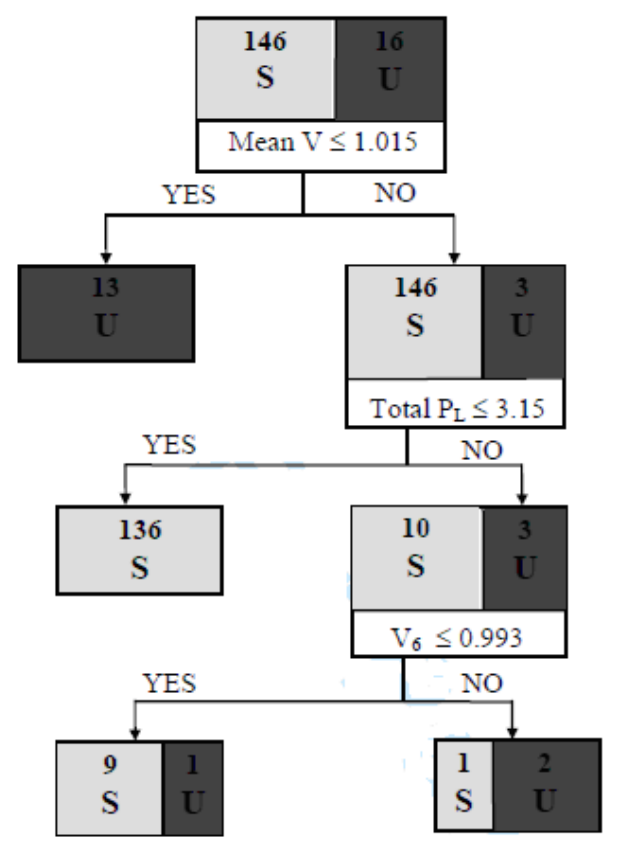

Mean $\mathrm{V}=$ mean of buses voltage

Total $\mathrm{P}_{\mathrm{L}}=$ total active power load

$\mathrm{V}_{6}=$ voltage at bus 6 .

Fig. 4: The Best Results Obtained using Decision Tree with Type I Attributes 


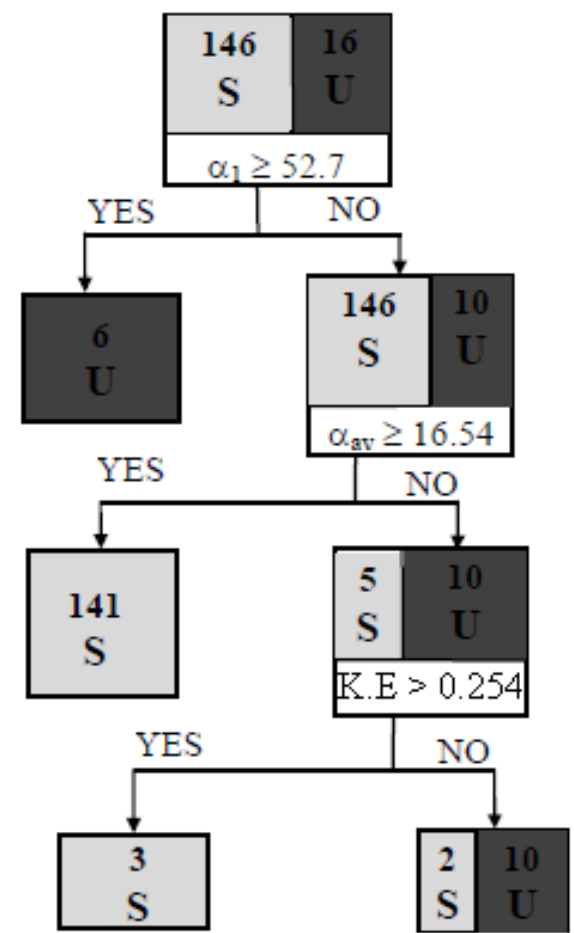

$\alpha_{1}=$ acceleration of $G_{3}$ at instant of fault inception

$\alpha_{\mathrm{av}}=$ average acceleration of $\mathrm{G}_{3}$

K.E. $=$ kinetic energy of $\mathrm{G}_{3}$ at instant of fault clearing

Fig. 5: The Best Results Obtained using Decision Tree with Type II Attributes

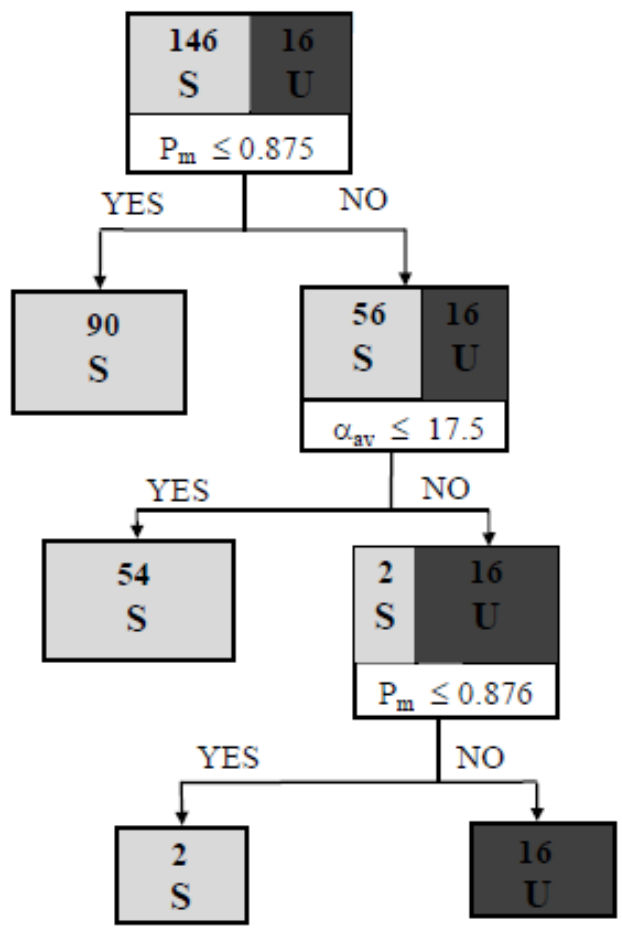

$\mathrm{P}_{\mathrm{M}}=$ mechanical input power of $\mathrm{G}_{3}$

Fig. 6: The Best Results Obtained using Decision Tree with Type III Attributes

\subsection{Testing the Decision Trees Approach}

The last step in the approach is the generalization process by which a complete verification of the capabilities of the approach in detecting the class of unknown samples is performed. The generalization ability is best stated in probabilistic terms as the probability of correct classification. It can serve as an index of satisfactory performance of the classification in unknown situations. This step is conducted by testing the approach using an adequate test set.

The samples of the test set should cover a wide spectrum of operating conditions and contingencies that the machine under study may be subjected to. The generation of samples for the test set is performed in a similar way to the training set. The test set is generated at six fault locations with four different load levels $(1.75,1.25,0.75,0.25)$ p.u. for each of the three loads. This produces 384 samples.

Reliability testing of DT is made by comparing the results against that obtained from the transient stability program. Table (1) shows the percentage misclassification with different attribute types.

Table 1: Performance Evaluation of the Approach

\begin{tabular}{|c|c|c|}
\hline $\begin{array}{c}\text { Attribute } \\
\text { Type }\end{array}$ & $\begin{array}{c}\text { Misclassification \% } \\
\text { In DT Building }\end{array}$ & $\begin{array}{c}\text { Misclassification \% in } \\
\text { Unseen Cases }\end{array}$ \\
\hline Type I & 1.85 & 3.13 (12 samples $)$ \\
\hline Type II & 1.23 & 1.56 (6 samples) \\
\hline Type III & 0.0 & 0.78 (3 samples) \\
\hline
\end{tabular}

The percentage error of classification by the DT with fault-on attributes is found to be lower than the pre-fault attributes. Also, it is concluded that the reliability of the DT generated by Type III attributes is better than the DTs generated using Types I and II attributes.

\section{Application of Fuzzy Concepts to the Transient Stability Problem}

The same training and testing sets used for the IIR approach are also used for the application of the fuzzy concepts to the TSA problem.

\subsection{Selection of Most Important Variables}

Only three features are chosen using the Single Ranking method based on previous experience of applying a pattern recognition technique to the same problem [5]. The first is the pre-fault loading of the generator or the mechanical input power 'Pm'. It is known that the higher the loading of generator, the higher the risk of instability. The second feature is the generator kinetic energy deviation K.E or ' $1 / 2 \mathrm{M} \omega^{2}$, at $\mathrm{t}=\mathrm{T}_{\mathrm{cl}}+$. 
The third feature is the average acceleration during fault ' $\alpha_{\mathrm{av}}$ '. It is the average value of the two rotor angular accelerations $\alpha 1$ at $\mathrm{t}=\mathrm{T}_{\mathrm{f}}^{+}$and $\alpha_{2}$ at $\mathrm{t}=\mathrm{T}_{\mathrm{cl}}{ }^{+}$.

$\mathrm{Tf}$ is the instant of fault and $\mathrm{T}_{\mathrm{cl}}$ is the instant of fault clearing. 'Pm' is a direct outcome of the load flow results. The other two features are determined from a transient stability study using the second-order model of the machine.

\subsection{Generation of Fuzzy Rules}

The procedure of generating fuzzy rules consists of two phases: partitioning the pattern space into fuzzy subspaces, then inducing a fuzzy rule for each subspace. So, the three previous variables, which are the inputs of the proposed fuzzy system, are divided as subsets as follows:

1. Loading condition or mechanical input power of the machine (Light, Normal, Heavy).

2. Kinetic Energy deviation of the generator at the instant of fault clearing (Low, High).

3. Average acceleration of machine (Very Small, Small, Medium, Large, Very Large).

The fuzzy subsets of the three normalized input variables relations against their degrees of membership are shown in Figs. (7), (8) and (9).

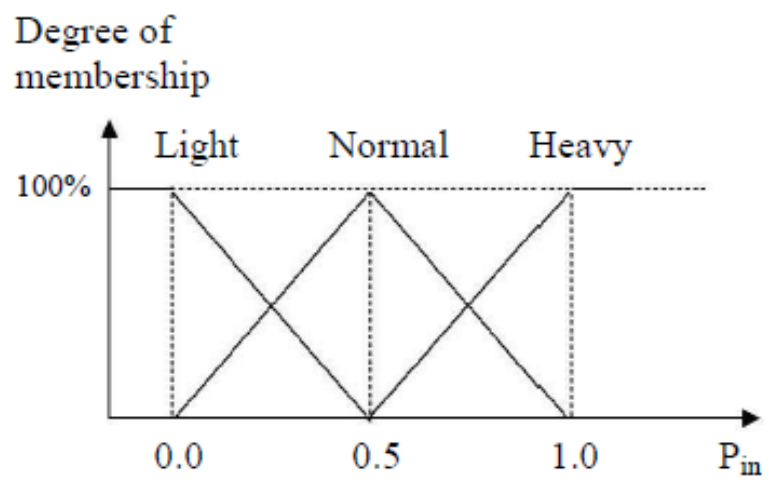

Fig. 7: Fuzzy Subsets for the Loading Condition

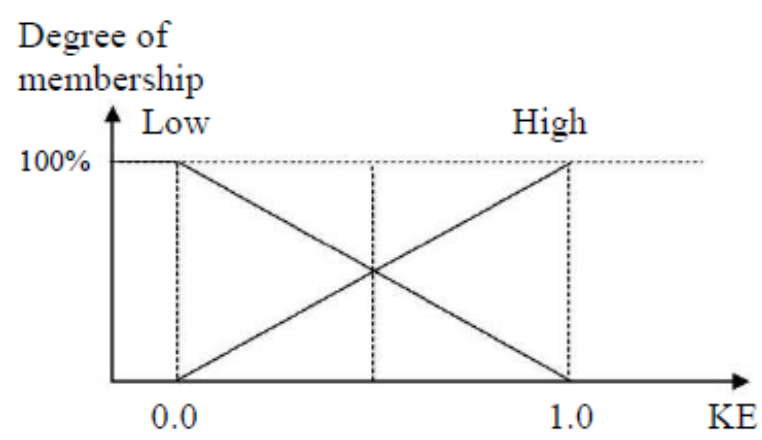

Fig. 8: Fuzzy Subsets for the Kinetic Energy

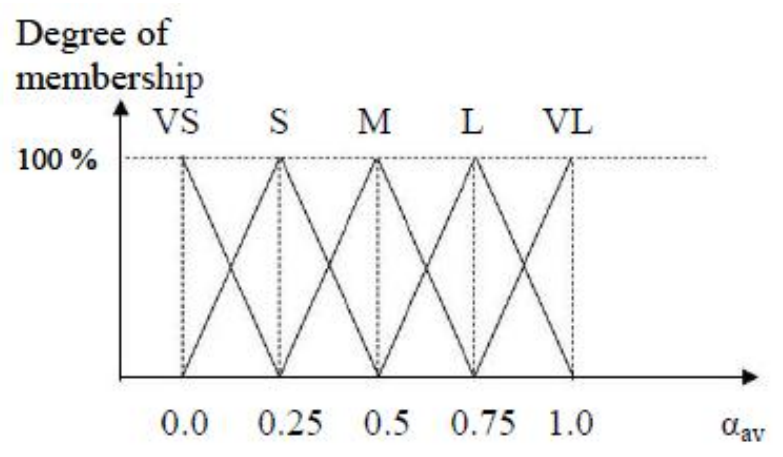

Fig. 9: Fuzzy Subsets for the Average Acceleration

After generating the training set, two different schemes are designed using the proposed fuzzy classification technique. The first scheme uses the three input variables as follows: (Pm: 3 subsets, K.E: 2 subsets, $\alpha_{\mathrm{av}}: 3$ subsets). The simulation results are used as the database to build the rule-base of the fuzzy classifier system. Tables $2 \& 3$ explain the rule base for the stability classification of the training set using the first fuzzy classifier scheme.

Table 2: Rule Base of the First Scheme (K.E is low)

\begin{tabular}{|c|c|c|c|}
\hline $\mathbf{P}_{\mathbf{m}}$ & Light & Normal & Heavy \\
\hline Small & Stable & Stable & \\
\hline Medium & Stable & Stable & Stable \\
\hline Large & ---- & Unstable & Unstable \\
\hline
\end{tabular}

Table 3: Rule Base of the First Scheme (K.E is High)

\begin{tabular}{|c|c|c|c|}
\hline $\mathbf{P}_{\mathbf{m}}$ & Light & Normal & Heavy \\
\hline Small & ---- & ---- & --- \\
\hline Medium & ---- & Stable & Stable \\
\hline Large & ---- & Unstable & Unstable \\
\hline
\end{tabular}

Table 4: Rule Base of the Second Scheme

\begin{tabular}{|c|c|c|c|}
\hline $\mathbf{P}_{\mathbf{m}}$ & Light & Normal & Heavy \\
\hline Very Small & Stable & Stable & \\
\hline Small & Stable & Stable & Stable \\
\hline Medium & --- & Stable & Stable \\
\hline Large & --- & Unstable & Unstable \\
\hline Very Large & ---- & & Unstable \\
\hline
\end{tabular}

Using the samples of the training set, the previous scheme presents an unacceptable classification performance. Six of the sixteen unstable samples are classified as stable with a high misclassification percentage $(37.5 \%)$. Also, it is concluded from Tables (2) and (3) the insignificance effect of the kinetic energy as an effective feature. So, the second scheme is designed as follows: ( $\mathrm{P}_{\mathrm{m}}$ : 3 subsets, $\alpha_{\mathrm{av}}$ : 5 subsets). 
Table (4) shows the rule base for the stability classification of the training set using the second fuzzy classifier scheme.

The classification performance of the second fuzzy logic classifier was very good. The total number of misclassified samples is 2 out of 162 and all the unstable samples are correctly classified. In order to test the proposed fuzzy classifier system, a test set comprising of 384 samples was generated by the same way as the training set. Table (5) shows the performance of the two different fuzzy classifier schemes using both the training and test sets.

Table 5: Results of Fuzzy Classification Based Schemes

\begin{tabular}{|l|c|c|}
\hline \multicolumn{1}{|c|}{ Fuzzy Scheme Used } & Misclassification of Training Patterns (out of 162) & Misclassification of Testing Patterns (out of 384) \\
\hline Scheme (1) & $6 / 16$ unstable, $37.5 \%$ & $4 / 40$ unstable, $10 \%$ \\
$\left(\mathrm{P}_{\mathrm{m}}: 3\right.$ subsets, K.E.: 2 & $0 / 14$ stable, $0 \%$ & $8 / 344$ stable, $203 \%$ \\
Subsets, $\alpha_{\mathrm{av}}: 3$ subsets) & $6 / 162$ total, $3.7 \%$ & $12 / 384$ total, $3.5 \%$ \\
\hline Scheme 2 & $0 / 16$ unstable, $0 \%$ & $1 / 40$ unstable, $2.5 \%$ \\
$\left(\mathrm{P}_{\mathrm{m}}: 3\right.$ subsets, & $2 / 146$ stable, $1.4 \%$ & $3 / 344$ stable, $0.8 \%$ \\
$\alpha_{\mathrm{av}}: 3$ subsets) & $2 / 162$ total, $1.2 \%$ & $4 / 384$ total, $1.04 \%$ \\
\hline
\end{tabular}

\subsection{Discussion}

The first author has presented different AI techniques for solving the TSA problem [5, 7] beside the work in this paper. It is concluded that the correct classification percentage is almost the same for the different schemes (ranging from 97 - 99\%). The common advantages of all the AI techniques used are:

1- They do not need prior knowledge of the system and/or any mathematical model of the machines.

2- The prediction of the transient stability in real time is based on simple computation.

3- These schemes are inherently adaptive since they are trained using different fault locations and load levels.

The difficulty in designing the classifier may limit the use of the pattern recognition technique. The DT and fuzzy logic schemes are characterized rather than the pattern recognition and neural networks methodologies by their simplicity since they used just only two features $\left(\mathrm{P}_{\mathrm{m}}, \alpha_{\mathrm{av}}\right)$. Consequently, it is just needed to measure the values of the active power of the machine before fault, at the instant of fault inception and at the instant of fault clearing to calculate the two features.

\section{Conclusion}

Two AI techniques (IIR and fuzzy logic) for transient stability assessment are presented. The successful identification of stable and unstable classes is significantly dependent on the chosen attributes. For example, voltage magnitudes usually vary in a narrow band near rated values and are unlikely to be sufficiently discriminatory. Also, voltage angles are not normally available in actual systems. So, the chosen attributes should assess the properties of each class and should fulfill discrimination, reliability and independence.
The significant reduction in misclassification error demonstrates that the implicit assumption of regarding the sufficiency of steady state variables for presentation of stable and unstable classes is unjustified. This means that selection of only steady state variables as input attributes would result in overlapping of stable and unstable classes and consequently some level of error. This error cannot be reduced with any refinement in the process of feature extraction.

The introduction of transient variables as input attributes guarantees a low error which is acceptable from a practical point of view. Since transient variables are closely related to the phenomenon of stability, it is expected that the misclassification error would not change significantly with the size of the system.

Unlike most of the existing methods, the two approaches do not impose any tradeoff between modeling sophistication and simplicity in use. In other words, modeling and on-line capabilities are almost independent issues. The computational burden linked to modeling refinements is to be faced during the off-line phase only, while building DTs. The on-line use of those DTs is scarcely to influence the computation times.

The degree of accuracy of the fuzzy rule-based classifier scheme is high and computation is fast enough for the program to be used for on-line transient stability assessment. Compared with other AI approaches used for the same problem, this scheme reveals simplicity, speed and comparable correct classification performance.

Future extension of the work is the application of the two proposed techniques to large-scale power systems.

\section{References}

[1] H. W. Dommel and N. Sato, "Fast Transient Stability Solutions", IEEE Transactions on Power 
Apparatus and Systems, Vol. PAS-91, July/August 1972, pp. 1643-1650.

[2] A. A. Fouad, and S. E. Stanton, "Transient Stability of a Multi-machine Evaluation Power System", Part I, II , IEEE Transactions on Power Apparatus and Systems, Vol. 100, No. 7, July 1981, pp. 3408-3424.

[3] R. Billiniton and P. R. S. Kuruganty, "Probabilistic Assessment of Transient Stability in a Practical Multimachine System", IEEE Transactions on Power Apparatus and Systems, Vol. PAS-100, No. 7, July 1981, pp. 3634-3641.

[4] H. Hakimmashhadi, "Fast Transient Security Assessment", Ph. D. Thesis, Purdue University, Indiana, USA, August 1982.

[5] A. M. El-Arabaty, H. E. A. Talaat, M. M. Mansour, and A. Y. Abdelaziz, "Out-of-step Detection Based on Pattern Recognition", International Journal of Electrical Power \& Energy Systems, Vol. 16, No. 4, August 1994, pp. 269-275.

[6] E. Hobson and G. N. Allen, "Effectiveness of Artificial Neural Networks for First Swing Stability Determination of Practical Systems", IEEE Transactions on Power Systems, Vol. 9, No. 2, May 1994, pp. 1062-1068.

[7] A. Y. Abdelaziz, M. R. Irving, A. M. El-Arabaty and M. M. Mansour, "Out-of-step Prediction Based on Artificial Neural Networks", Electrical Power Systems Research, Vol.34, No. 2, August 1995, pp. 135-142.

[8] L. Wehenkel, Th. Van Cutsem and M. RibbensPavalla, "An Artificial Intelligence Framework for On-line Transient Stability Assessment of Power Systems", IEEETransactions on Power Systems,Vol. 4, No. 2, May 1989, pp. 789-800.

[9] L. Wehenkel, M. Ribbens-Pavalla, E. Euxibie and B. Heilbronn, "Decision Trees Based Transient Stability Method: A case Study", IEEE Transactions on Power Systems, Vol. 9, No.1, February 1994, pp. 459-468.

[10] S. Rovnyak, S. Kretsinger, J. Thorp and D. Brown, "Decision Trees for Real-Time Transient Stability Prediction", IEEE Transactions on Power Systems, Vol. 9, No. 3, August1994, pp. 1417-1426.

[11] K. Yabe, K. Yoshida, N. Rostam Kalai, P. E. Nielsen and D. J. Leonard, "The New Concept of an Artificial Intelligent Based Smart Relay", International Journal of Engineering Intelligent Systems, Vol. 2, No. 4, December 1994, pp. 213 221.

[12] S. Rovnyak, C. Taylor and Y. Sheng, "Decision Trees Using Apparent Resistance to Detect Impending Loss of Synchronism", IEEE Transactions on Power Delivery, Vol. 15, No.4, October 2000, pp. 1157 - 1162.
[13] S. P. Teeuwsen, I. Erlich, M. El-Sharkawi and U. Bachmann, "Genetic Algorithm and Decision Tree-Based Oscillatory Stability Assessment, IEEE Transactions on Power Systems, Vol. 21, No. 2, May 2006, pp. 746 - 753.

[14] J. L. Souflis, A. V. Machias and B. C. Papadias, "An Application of Fuzzy Concepts to Transient Stability Evaluation", IEEE Transactions on Power Systems, Vol. 4, No. 3, August 1989, pp. 1003 1009.

[15] L. Lai, J. T. Ma, M. Almoncid and Y. Yang, "Online Multi-machine Power System Stability Assessment with Fuzzy Logic Classifier", Proceedings of the 30th Universities Power Engineering Conference, UPEC'95, Greenwich, September 1995, pp. 172-175

[16] H. E. A. Talaat, "Predictive Out-of-Step Relaying using Fuzzy Rule-Based Classification", Electric Power Systems Research, Vol. 48, No. 3, January 1999, pp. 143-149.

[17] C. Liu, S. Tsay, Y. Wang and M. Su, "NeuroFuzzy Approach to Real-Time Transient Stability Prediction based on Synchronized Phasor Measurements", Electric Power Systems Research, Vol. 49, No. 2, March 1999, pp. 123-127.

[18] W. P. Ferreira, M. d. C. G. Silveira, A. P. Lotufo, Carlos. R. Minussi, "Transient Stability Analysis of Electric Energy Systems via a Fuzzy ARTARTMAP Neural Network", Electric Power Systems Research, Vol. 76, No. 6, April 2006, pp. 466-475.

[19] W. Li, J. Zhou, K. Xie and X. Xiong, "Power System Risk Assessment Using a Hybrid Method of Fuzzy Set and Monte Carlo Simulation”, IEEE Transactions on Power Systems, Vol. 23, No. 2, May 2008, pp. 336 - 343.

[20] R. S. Michalski, J. G. Carbonell and T. M. Mitchell, "Machine Learning: An Artificial Intelligence Approach", Tioga Publishing Company, Palo Alto, 1983.

[21] J. Mamoh, X. W. Ma and K. Tomsovic, "Overview and Literature Survey of Fuzzy Set Theory in Power Systems", IEEE Transactions on Power Systems, Vol. 10, No. 3, August 1995, pp. 16761690.

[22] E. Cox, "The fuzzy System Handbook: A Practitioner's Guide to Building, Using, and Maintaining Fuzzy Systems", Academic Press, USA, 1994.

[23] P. M. Anderson and A. A. Fouad, "Power System Control and Stability", Iowa State University Press, Iowa, 1977. 


\section{Authors' Profiles}

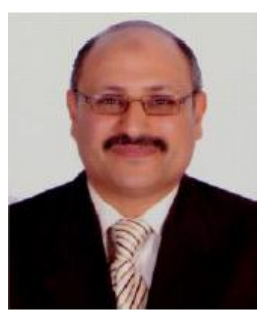

Almoataz Y. Abdelaziz was born in Cairo, Egypt, on 1963. He received the B. Sc. and $M$. Sc. degrees in electrical engineering from Ain Shams University, Cairo, Egypt in 1985, 1990 respectively and the $\mathrm{Ph}$. D. degree in electrical engineering according to the channel system between Ain Shams University, Egypt and Brunel University, England in 1996. He is currently a professor of electrical power engineering in Ain Shams University.

His research interests include the applications of artificial intelligence to power systems and protection and new optimization techniques in power systems operation and planning. He has authored or coauthored more than 140 refereed journal and conference papers. Dr. Abdelaziz is a member of the editorial board and a reviewer of technical papers in several international journals. He is also a member in IEEE, IET and the Egyptian Sub-Committees of IEC and CIGRE'.

Dr. Abdelaziz has been awarded Ain Shams University Prize for distinct researches in 2002 and for international publishing in 2010, 2012.

Maher A. El-Dessouki Received his Ph.D. degree from Warsaw University of technology in 1994 in dynamic study of power systems considering electrical machines as dynamic loads. Now, he is an assistant professor in the Department of Electrical Power and Machines, Faculty of Engineering, Ain Shams University, Cairo, Egypt.

His research interests include modeling, simulation, control of electrical machines, power systems dynamics, power system stability, power system reconstruction, design of novel electrical machines, new techniques in power system distribution, use of the artificial intelligent in the control of both the electrical machines and power systems. He supervised many research projects. He teaches many courses of electrical machines and power system inside and outside Egypt in different universities.

Mr. El-Dessouki is a member of the Association of Energy engineers (AEE). 